\title{
Wavelet-Fourier analysis of electric signal disturbances
}

\author{
$\frac{\text { Juan-Carlos Montaño }}{1}^{1}$, Dolores Borrás $^{2}, \frac{\text { Juan-Carlos Bravo }^{2}}{\text { and } \text { Jaime Gutiérrez }^{4}}$, Manuel Castilla $^{2}, \underline{\text { Antonio López }}^{3}$ \\ ${ }^{1}$ Consejo Superior de Investigaciones Científicas (CSIC), IRNAS. \\ Reina Mercedes, 10 .41012-Sevilla, Spain. montano@irnase.csic.es \\ ${ }^{2}$ Dpto. Ingeniería Eléctrica. Escuela Universitaria Politécnica \\ Virgen de Africa, 7, 41011-Sevilla, Spain. \\ Tel.:+34 954552831,(2814),(2847), fax:+34 954282777, \\ carlos_bravo@us.es borras@us.es castilla@us.es \\ ${ }^{3}$ Dpto. Tecnología Electrónica. Escuela Universitaria Politécnica \\ Virgen de Africa, 7, 41011-Sevilla, Spain. alojeda@us.es \\ ${ }^{3}$ Dpto. Física Aplicada III. Escuela Superior de Ingenieros \\ Avda. de los Descubrimientos s/n, 41092-Sevilla, Spain. jaiben@us.es
}

\begin{abstract}
The Fourier transform usually has been used in the past for analysis of stationary and periodic signals. Its interest is the knowledge of spectral components existing in a waveform; it doesn't matter the moment where they happen. However, when the time localization of the spectral components is needed, the Wavelet Transform (WT) can be used to obtain the optimal timefrequency representation of the signal. In this paper, the joint wavelet-Fourier transform has been proposed for detecting, analyzing and compacting electrical disturbances. Finally, results of experiments have been included.
\end{abstract}

Keywords: Wavelets, Fourier transform, power quality, harmonic distortion, transients.

\section{Introduction}

Waveform evaluation consists of both spectrum and transient analysis. There are a few algorithms of voltage and current waveform analysis. The evaluation of the algorithm efficiency involves the estimation of its accuracy combined with the required computational power. Unfortunately, the latter has an important meaning in case of real-time metering. However, implementing new algorithms is not always necessary. Sometimes it is enough to apply broadly known tools in a new way to achieve better results.

In order to find out the sources and causes of harmonic distortion, one can detect and localize those disturbances for further classification. Software procedures have been developed for this purpose, such as the fast Fourier transform (FFT), due to its low computational complexity [1].

However, transients are commonly analyzed by means of discrete wavelet transform (DWT), apart from some algorithms based on Fourier transform like short-time Fourier transform (STFT) [1-4].

In this environment, power quality analysis strategies have usually been divided into those that address steadystate concerns, such as harmonic distortion, and transient concerns, like those resulting from faults or switching transient. Technique such as Fourier spectral analysis is often applied to steady-state events while wavelets, classical transient analysis, computer modelling are traditionally used for transient events.

The goal of this work is the use of the wavelet analysis as well as the Fourier analysis for a generic signal (voltage or current signals), in transient or steady state situations, for detecting and classifying power quality events. The use of DWT for compressing the disturbed signal before applying FFT permits to get a higher compression rate and a faster signal processing than that obtained using only the conventional DWT/FFT approach.

The proposed method is based on both, the wavelet analysis and the Fourier analysis, as a new hybrid way to study disturbed signals. So, the original signal $f(t)$ can be represented approximately as a superposition of scaling functions $\varphi_{J 0, k}(t)$, and wavelets $\psi_{j, k}(t)[4]$.

$$
f(t)=\sum_{k=0}^{2^{J_{0}-1}} a_{J_{0}, k} \varphi_{J_{0}, k}(t)+\sum_{j=J_{0}}^{J-1} \sum_{k=0}^{2^{j}-1} d_{j, k} \psi_{j, k}(t)
$$

where the approximation is truncated at $j=J-1$, and sets $a_{J 0, k}$ and $d_{j, k}$ are the DWT of the signal $f(t)$, which can be calculated by 


$$
\begin{aligned}
& a_{J_{0}, k}=\sum_{m} h(m-2 k) a_{J_{0}+1, m} \\
& d_{j, k}=\sum_{m} g(m-2 k) d_{j+1, m}
\end{aligned}
$$

In expression (1), the first sum is a coarse representation of $f(t)$, where $f(t)$ has been replaced by a linear combination of $2^{J_{0}}$ translations of the scaling function $\varphi_{J 0,0}$. The remaining terms are the detailed representation. For each $j$ level, $2^{j}$ translations of the wavelet $\psi_{j, 0}$ are added to obtain a more detailed representation of $f(t)$.

The wavelet approach is more suitable than the usual Fourier Transform, in those cases in which we are interested in getting good time resolution at the high frequency range for non-stationary signals. It can be viewed as a transformation from the time domain to the time-frequency (scale) domain. In wavelet analysis, the scale we use to look at the data plays a special role.

The Multiresolution Signal Decomposition algorithm is used in this work [4], according to diagram of Fig. 1 and nomenclature of (1).

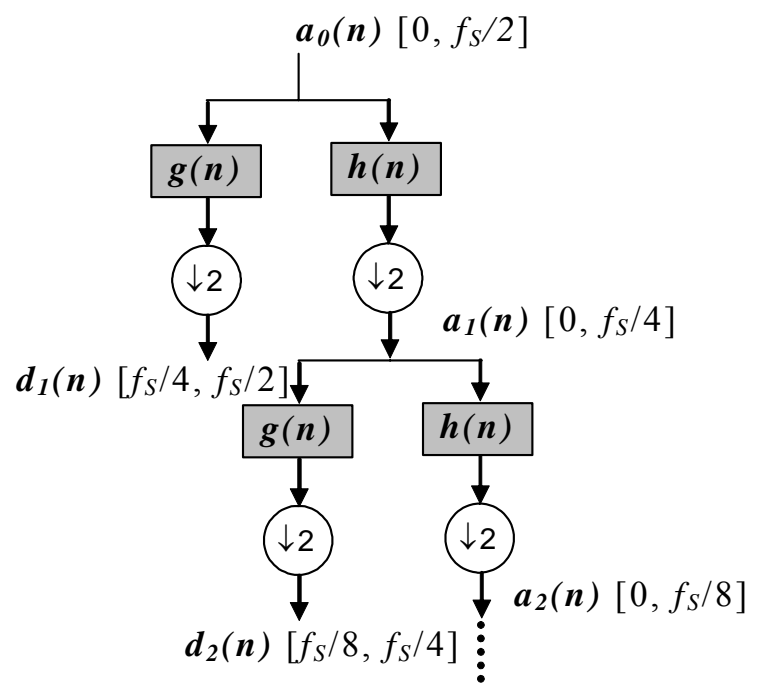

Fig.1: Multiresolution signal decomposition. Sample of analysis filter banks $h$ : low-pass filter coefficients. g: high-pass filter coefficients.

\section{Power System Disturbances: detection, location and discrimination}

\section{A. The method}

We propose an effective method (Fig. 2) to detect, analyze and discriminate power system disturbances using joint wavelet-Fourier decomposition. Our main goal is to perform the desired separated extraction of permanent events (harmonic content), and transient and notching events (random disturbances, such as sags, swell, oscillatory transient, notching, etc.) for a given signal $f(t)$. The DWT (Daubechies family Db4) is applied to a digitized function with $N$ samples per monitoring window. Firstly, the DWT analysis comprises the first step of the MRA method (one scale) for disturbance detection.

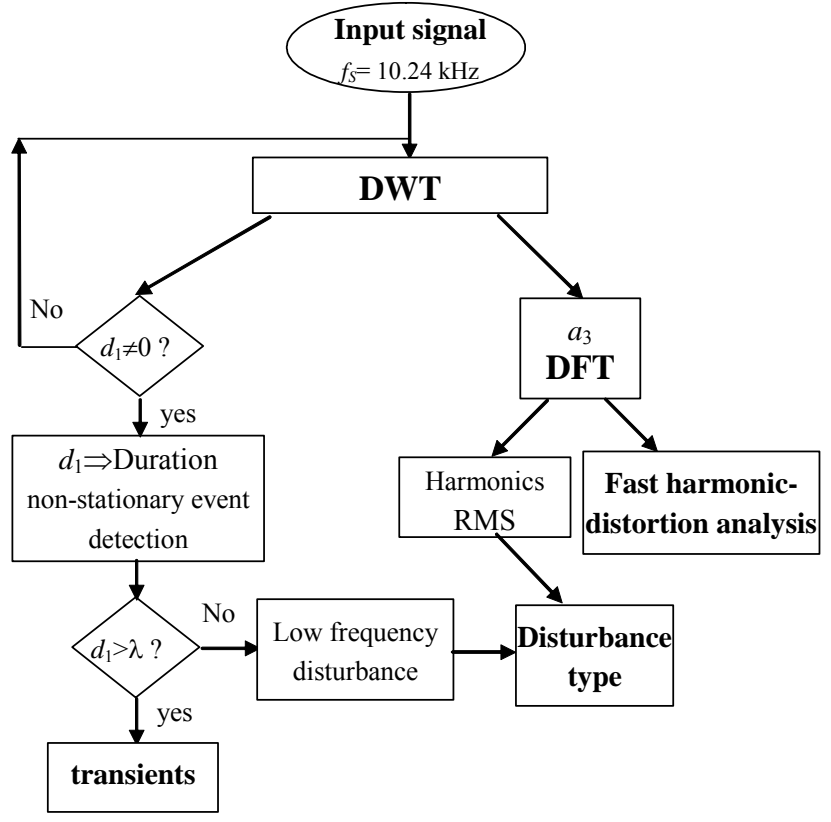

Fig. 2: Flowchart of the proposed method

1) Detection and discrimination

The method fundamentals, in short, consist of a few simple assumptions.

- Harmonics and interharmonics analysis should typically cover a frequency band up to $6 \mathrm{kHz}$ [6].

- Transient and notching analysis should at least cover a frequency band above $40 \mathrm{kHz}$ [7].

- Wavelet coefficients for each decomposition layer contain relevant frequency information [4].

The information about the position and length of a power system disturbance is obtained from the detail coefficient $d_{1}(n)$. This signal $d_{1}(n)$ is non-zero when a transient disturbance exists.

Two processes occur at the same time; the first one is the disturbance detection and the other one is the input signal compression applying the DFT over the suitable band. Next, a parallel process begins the DFT analysis for stationary events and, using the $d_{1}$ signal, the separation of the disturbance in non-stationary state based upon the wavelet decomposition characteristics.

Next, a threshold process begins, based at maximum absolute value of $d_{1}(n)$ signal. The initial threshold is expressed by

$$
\lambda=\eta \cdot \max \left|a_{0}(n)\right|
$$

where $a_{0}(n)$ is the sampled input signal and $\eta$ is a parameter that varies between 0.001 and 0.01 . Thus, for $\eta=0.005$, the threshold $\lambda$ is $0.5 \%$ of the maximum value of the $d_{1}$ signal. A process of comparison between each point of the $d_{1}(n)$ signal and the $\lambda$ value begins. If any $d_{1}(n)$ point is greater than $\lambda$ a transient event occurs; else a non-stationary low frequency disturbance is present.

When a low frequency disturbance is detected, the DFT process is needed to calculate the RMS value of the signal contained in the measurement window to identify the disturbance. 
2) Harmonic analysis and classification

The $a_{3}$ coefficient signal is the input data for the DFT analysis. The goal is a faster DFT algorithm because it works over the $1 / 8$ size of the input signal.

The RMS value of the harmonic analysis for this compressed signal enables to discriminate if the disturbance is sag, interruption or swell. If the RMS values of the input signal and the fundamental component are equal and the RMS value of $d_{1}(n)$ is above a threshold, a transient disturbance is detected.

\section{B. Simulation results using Mathcad}

In order to illustrate these ideas, the Mathcad13 $\mathbb{}$ program is used to calculate the DWT and DFT for a digitized voltage-signal within a window width equal to exactly ten periods of the fundamental period for $50-\mathrm{Hz}$ systems (200 ms). It corresponds to 204.8 sample points per period, sampled at $\omega_{\mathrm{S}}=10.24 \mathrm{kHz}$ during total interval $T$, equivalent to ten cycles. This rectangular window size is recommended by Std. 61000-4-7, IEC [8].

In our simulations a steady-state disturbance consisting of harmonic distortion was considered. The basis harmonic-content consists of the fundamental component at $50-\mathrm{Hz}$, plus the 5th and 7th harmonic components, with rms voltage amplitudes: Vnom, (6\%)Vnom, and (4\%)Vnom, respectively, where Vnom $=1 / \sqrt{ } 2$.

Superimposed to this steady-state event, we have considered two cases of transient disturbances: an oscillatory transient (Case 1), and a momentary voltagesag (Case 2) represented by a modulating trapezoidal waveform whose effect is to decrease by a 0.2 factor the signal magnitude over an interval of 5 cycles. We have performed numerical simulations with these input signals (Figs. 3 and 4).

For our purpose, the DWT (Bspline 3,7) is applied firstly to the digitized function of Case 1 and Case 2 within a 10 cycles window of 2048 samples. The max number of levels is the power of two equal to the number of samples, (in our case $J=11$ ). For these two functions and $J=5$, the results of the "multiresolution analysis" are shown in Figs. 5 and 6 , respectively, where six coefficient signals $a_{0}, d_{0}, . ., d_{J}$, correspond to the respective scales (band of frequencies): $j=0,1, \ldots, 5$.

A detection process is derived from the assessment of rms values of $d_{j}$ coefficient signals and the fundamental component to identify the instants at which the disturbance appears and vanishes.

By applying the DFT algorithm to the coefficients signal $a_{0}(n)$, in Case 1 , we find out the input signal approximately free of the harmonic content and high frequency components due to the momentary oscillatory transient. Fig. 5 shows the distribution of the harmonic content within the scales 1 to 5 .

The exposed procedure also improves the speed of the Fourier analysis due to a $1 / 8$ reduction of the input data since the coefficients the coarse signal $a_{3}(t)$ contains 130 samples instead of 2048 samples of the input signal.
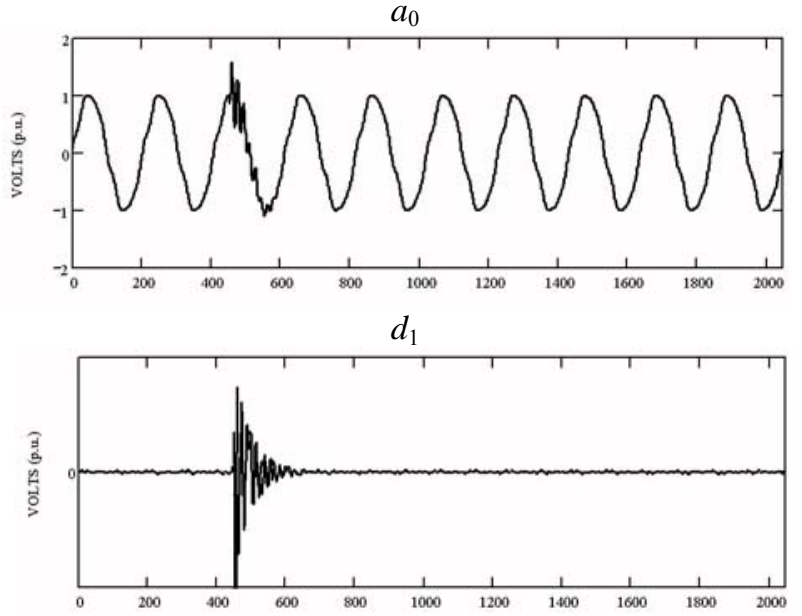

Fig.3. Simulated case of voltage disturbances: harmonics and oscillatory transient.

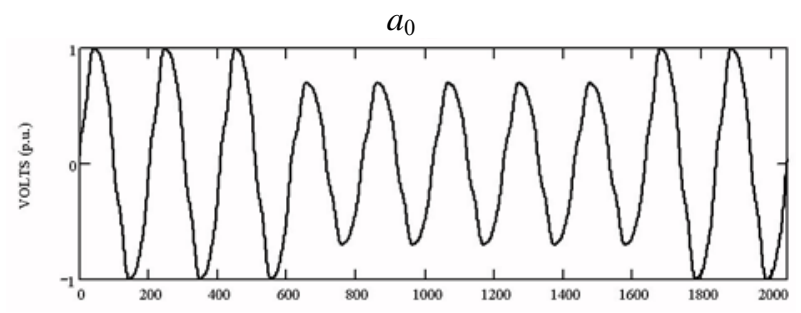

$d 1$

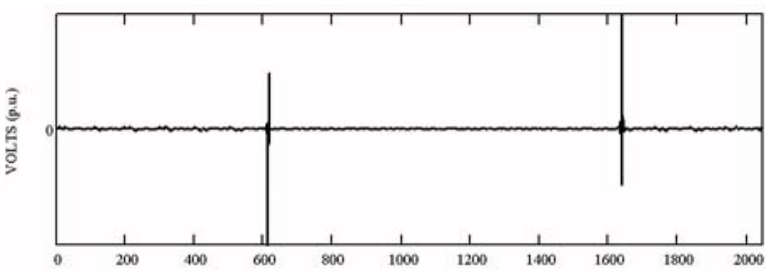

Fig.4. Simulated case of voltage disturbances: harmonics and momentary sag.

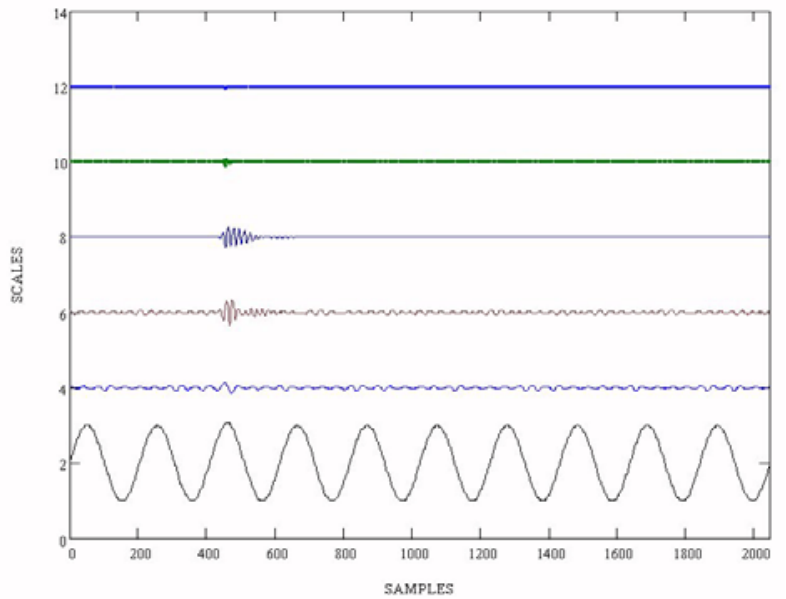

Fig. 5. Coefficient signals $a_{0}, d_{0}, . ., d_{5}$, corresponding to the input signal shown in Fig. 3 , for scales $j=0,1, \ldots, 5$. 


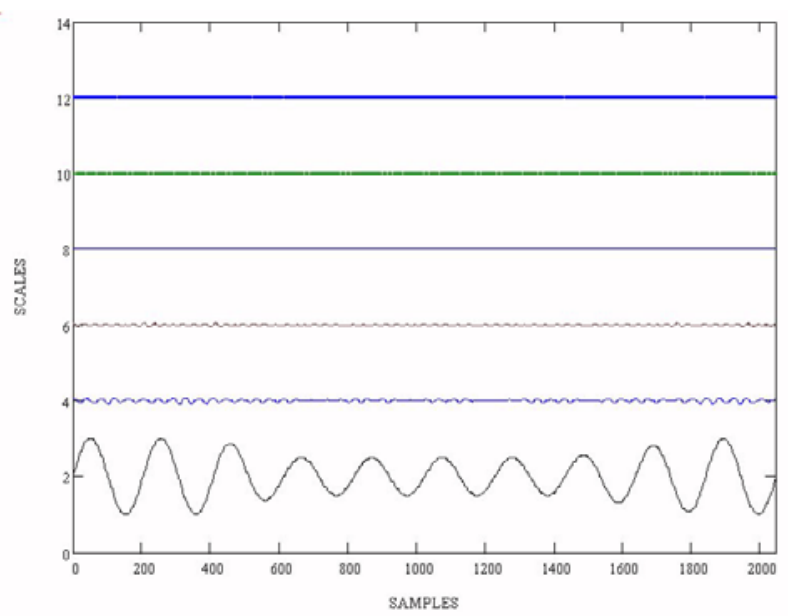

Fig. 6. Coefficient signals $a_{0}, d_{0}, . ., d_{5}$, corresponding to the input signal shown in Fig. 4 , for scales $j=0,1, \ldots, 5$.

For Case 2 (Fig. 6) we find out the harmonic content of the input signal disseminated within the 1 to 5 scales, and signal $a_{0}(n)$ free of harmonic components but disturbed in amplitude due to the momentary voltage sag.

\section{Conclusions}

In this work we have developed a new approach to detect, classify and analyze various types of power systems disturbances using a joint wavelets-Fourier method. It permits a faster DFT processing and an effective detection and classification of stationary and non-stationary events.

DWT is the fastest transform for detection and compression purpose in non-stationary state and the DFT is the best for harmonics location. Both methods (DFT and DWT) have their own limitations in signal disturbance analysis. Our procedure extracts the best of each other for an optimal detection and classification of several disturbances. So, steady-state and transient-state disturbances are detected, analyzed, and classified.

\section{References}

[1] L. Angrisani, P. Daponte, M. D’Apuzzo, and A. Testa, "A measurement method based on the wavelet transform for power quality analysis," IEEE Trans. Power Delivery, vol. 13, pp. 990-998, Oct. 1998.

[2] S.Mallat: "A theory for multiresolution signal decomposition: the wavelet representation." IEEE Trans. on Pattern Anal. and Mach. Intell., Vol. 11, pp.674-693.July 1989.

[3] S.Santoso, E.J.Powers and W.M. Grady: "Power quality disturbances data compression using wavelet transform methods." IEEE Trans. on Power Del. Vol. 12, no. 3. July 1997.

[4] D.Borrás, M. Castilla, N. Moreno and J.C. Montaño: "wavelet and neural structure: a new tool for diagnostic of power system disturbances" IEEE Trans. on Industry App. Vol. 37, no. 1. January 2001.
[5] J.C. Goswami and A.K. Chan, Fundamentals of Wavelets. Theory, Algorithms and Applications, John Wiley, Inc., NY, 1999.

[6] IEEE Recommended Practice on Monitoring Electric Power Quality IEEE 1159:1995.

[7] T. Tarasiuk and M. Szweda, "A few remarks about notching analysis - Case study," in Proc. IMEKO TC4 Int. Symp., 2004, pp. 504-509.

[8] General Guide on Harmonics and Interharmonics Measurements for Power Supply Systems and Equipment Connected Thereto, Std. 61000-4-7, IEC. 